\title{
On a Modification of Watson's Lemma
}

\section{F. Oberhettinger*}

(May 27, 1959)

\begin{abstract}
The method of steepest descents is extended to the case when a saddle point and a pole of arbitrary order are involved. An application to a problem in diffraction theory is demonstrated.
\end{abstract}

\section{Introduction}

The solution of numerous problems in electromagnetic and acoustic wave propagation theory is frequently given by an integral representation of the form

$$
\varphi(z)=\int_{C} w(v) e^{-z u(v)} d v
$$

Here $\varphi(z)$ denotes the desired property (for instance the electromagnetic or acoustic potential). The integration in (1) is taken along a curve $C$ in the complex $v$ plane along which $w(v)$ and $u(v)$ are defined. These function contain generally certain parameters which depend on the physical configuration under investigation. The parameter $z$ is usually related to the distance between some point of reference and the point of observation. A reduction of the integral (1) to an asymptotic series has to be carried out when approximate expressions for large $z$ are desired. The frequently used method of steepest descents $[3, \text { p. } 86]^{1}$ becomes invalid when one of the above mentioned parameters assumes a value for which a pole of $w(v)$ coincides with a saddle point of $u(v)$. Methods to cope with such cases have been worked out and discussed [1], [8], [9]. The former two give expansions which proceed in terms of hypergeometric functions besides a "leading term", while the latter consists of an error integral as leading term and an asymptotic series with inverse powers of $z$. It is shown here that the method given in [9], which involves a pole of the first order and a branch point of order 2 can be extended to the case involving a pole and a branch point of arbitrary order. The expansion for this case consists of a finite number (equal to the order of the pole) of terms involving Whittaker's functions and an asymptotic series with inverse powers of $z$.

\section{A Modification of Watson's Lemma}

It is assumed that the reduction of the contour integral (1) (for instance by the method of steepest descents) to one or several integrals of the Laplace

* Oregon State College, Department of Mathematics. (Invited paper.)

1 Figures in brackets indicate the literature references at the end of this paper. transform type

$$
f(z)=\int_{0}^{\infty} F(t) e^{-t z} d t
$$

has already been carried out and only this kind of integrals shall be considered. A theorem commonly referred to as Watson's lemma [2, p. 218] admits the asymptotic representation of $f(z)$ for large $z$ when the behavior of $F(t)$ for small $t$ is known. In its general form given by Doetsch [3, p. 48] and [4] one has the

Theorem: Let $F(t)$ be analytic in a sector $\alpha<$ arg $t<\beta$ of the complex $t$ plane with the possible exception of the origin, and let the behavior of $F(t)$ in this sector be

(a)

(b) $F(t) \approx \sum_{n=0}^{\infty} a_{n} t^{\alpha_{m}}, \quad-1<\operatorname{Re} \alpha_{0}<\operatorname{Re} \alpha_{1}<\cdots$

when $t \rightarrow 0$ in $\alpha<\arg t<\beta$.

The asymptotic expansion for $f(z)=\int_{0}^{\infty} F(t) e^{-z t} d t$ for large $z$ is then obtained by inserting the series (b) for $F(t)$ into $(2)$ and integrating term by term.

$$
f(z)=\int_{0}^{\infty} F(t) e^{-t z} d t \approx \sum_{n=0}^{\infty} a_{n} \Gamma\left(1+\alpha_{n}\right) z^{-\alpha_{n}{ }^{-1}}
$$

when $z \rightarrow \infty$ in $-\frac{\pi}{2}-\beta<$ arg $z<\frac{\pi}{2}-\alpha$.

The function $F(t)$ in (2) is now assumed to be of the form

$$
F(t)=t^{\alpha} g(t), \quad \text { Re } \alpha>-1,
$$

where the function $g(t)$ is analytic in the vicinity of $t=0$. In this case

with

$$
f(z)=\int_{0}^{\infty} t^{\alpha} g(t) e^{-t z} d t
$$

$$
g(t)=\sum_{n=0}^{\infty} a_{n} t^{n}, \quad a_{n}=\frac{1}{n !}\left[\frac{d^{n}}{d t^{n}} g(t)\right]_{t=0} .
$$




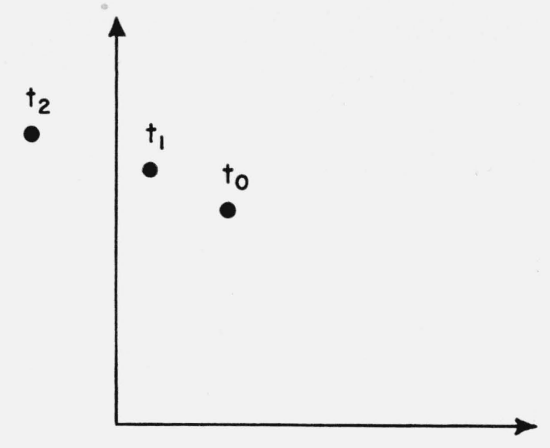

This series is convergent for $|t|<\left|t_{0}\right|$, where $t_{0}$ is that singular point of $g(t)$ which is closest to the origin. The other singular points are denoted by $t_{1}, t_{2},$. . . such that their moduli form a nondecreasing sequence. We assume that none of the singular points is on the positive real $t$-axis. The asymptotic expansion of (5) then becomes by (3) and (6)

$f(z)=\int_{0}^{\infty} t^{\alpha} g(t) e^{-t z} d t \approx \sum_{n=0}^{\infty} a_{n} \Gamma(\alpha+n+1) z^{-\alpha-n-1}$

for $z \rightarrow \infty$. The range of validity for $\arg z$ depends on the behavior of $g(t)$ according to (a) and (b) of the theorem quoted before. The case in which the singular point $t_{0}$ is a pole of order $m$, say, is considered now. The Laurent expansion of $g(t)$ in the vicinity of $t_{0}$ is then

$$
g(t)=b_{-m}\left(t-t_{0}\right)^{-m}+\ldots+b_{-1}\left(t-t_{0}\right)^{-1}+g^{*}(t)
$$

where

$$
g^{*}(t)=g(t)-b_{-m}\left(t-t_{0}\right)^{-m}-\ldots-b_{-1}\left(t-t_{0}\right)^{-1}
$$

is analytic for $|t|<\left|t_{1}\right|$ and consequently can be represented by the series

$$
g^{*}(t)=\sum_{n=0}^{\infty} c_{n} t^{n}, \quad c_{n}=\frac{1}{n !}\left[\frac{d^{n}}{d t^{n}} g^{*}(t)\right]_{t=0}, \quad|t|<\left|t_{1}\right| .
$$

It has to be remembered that in physical applications the function $g(t)$ in (4) contains generally certain parameters which depend on the configuration of the problem, and this may become such that the pole $t_{0}$ of $g(t)$ coincides with the origin $t=0$ (see section 3 ). In this case all the coefficients $a_{n}$ tend to infinity by (6) and (8) and the asymptotic series in (7) becomes useless although the integral in (7) may exist. It is therefore necessary to obtain an expansion which for $t_{0} \neq 0$ represents the integral (7) asymptotically for large $z$ and which for $t_{0}=0$ (if the integral exists) gives its correct value. Such an expansion can be obtained as follows: One inserts the representation (8) for $g(t)$ into $(7)$ and gets

$$
\begin{aligned}
& f(z)=\int_{0}^{\infty} t^{\alpha} g(t) e^{-t z} d t \\
& ==\sum_{k=1}^{m} b_{-k} \int_{0}^{\infty}\left(t-t_{0}\right)^{-k} t^{\alpha} e^{-t z} d t+\int_{0}^{\infty} t^{\alpha} g^{*}(t) e^{-t z} d t .
\end{aligned}
$$

But

$$
\begin{gathered}
\int_{0}^{\infty} t^{\alpha} e^{-t z}\left(t-t_{0}\right)^{-k} d t=\Gamma(1+\alpha)\left(-t_{0}\right)^{\frac{1}{2} \alpha-\frac{1}{2} k} z^{\frac{1}{2} k-\frac{1}{2} \alpha-1} \\
\times e^{-\frac{1}{2} t_{0} z} W_{-\frac{1}{2} k-\frac{1}{2} \alpha, \frac{1}{2} \alpha-\frac{1}{2} k+\frac{1}{2}}\left(-t_{0} z\right),
\end{gathered}
$$

by virtue of the integral representation for Whittaker's function [5, p. 274]. The integral

$$
\int_{0}^{\infty} t^{\alpha} g^{*}(t) e^{-t z} d t
$$

can be represented asymptotically for large $z$ by means of the power series expansion (10) (which is valid for $\left.|t|<\left|t_{1}\right|\right)$ and Watson's lemma. One obtains then finally

$$
\begin{aligned}
f(z) & =\int_{0}^{\infty} t^{\alpha} g(t) e^{-t z} d t \\
& \approx \sum_{n=0}^{\infty} c_{n} \Gamma(\alpha+n+1) z^{-\alpha-n-1}+\Gamma(1+\alpha)\left(-t_{0}\right)^{\frac{1}{2} \alpha} z^{-\frac{1}{2} \alpha-1} \\
& \times e^{-z t_{0}} \sum_{k=1}^{m} b_{-k}\left(\frac{z}{-t_{0}}\right)^{\frac{1}{2} k} W_{-\frac{1}{2} k-\frac{1}{2} \alpha, \frac{1}{2} \alpha-\frac{1}{2} k+\frac{1}{2}}\left(-z t_{0}\right) .
\end{aligned}
$$

The range of validity for $\arg z$ depends on the behavior of $g^{*}(t)$ according to the requirements (a) and (b) of the theorem quoted before. For certain combinations of $\alpha$ and $m$ (13) can reduce to a simpler expression, as for instance

$$
W_{k, \frac{1}{4}}(z)=2^{\frac{1}{4}-k} z^{\frac{1}{4}} D_{2 k-\frac{1}{2}}\left[(2 z)^{\frac{1}{2}}\right],
$$

(parabolic cylinder function)

$W_{-\frac{1}{4}, \frac{1}{4}}(z)=\pi^{\frac{1}{2}} z^{\frac{1}{4}} e^{\frac{1}{2} z} \operatorname{Erfc}\left(z^{\frac{1}{2}}\right)=z^{\frac{1}{4}} e^{\frac{1}{2} z} \int_{z}^{\infty} t^{-\frac{1}{2}} e^{-t} d t$

$W_{-\frac{1}{2}, 0}(z)=-z^{-\frac{1}{2}} e^{\frac{1}{2} z} \operatorname{Ei}(-z)=z^{-\frac{1}{2}} e^{\frac{1}{2} z} \int_{z}^{\infty} t^{-1} e^{-t} d t$

$W_{0, \mu}(z)=(z / \pi)^{\frac{1}{2}} K_{\mu}\left(\frac{1}{2} z\right),($ modified Bessel function $)$.

The case $\alpha=-\frac{1}{2}, m=1$ (branch point of order 2 and pole $t_{0}$ of the order 1 ) which leads to the special case (15) has been treated before [9]. For this case one obtains from (13) and (15) 


$$
\begin{aligned}
& f(z)=\int_{0}^{\infty} t^{-\frac{1}{2}} g(t) d t \approx \sum_{n=0}^{\infty} c_{n} \Gamma\left(n+\frac{1}{2}\right) z^{-n-\frac{1}{2}} \\
&+b_{-1} \pi\left(-t_{0}\right)^{-\frac{1}{2}} e^{-z t_{0}} \operatorname{Erfc}\left[\left(-z t_{0}\right)^{\frac{1}{2}}\right] .
\end{aligned}
$$

The coefficients $c_{n}$ according to (9) and (10) are defined by

$$
g(t)-b_{-1}\left(t-t_{0}\right)^{-1}=\sum_{n=0}^{\infty} c_{n} t^{n},
$$

or

$$
c_{n}=\frac{1}{n !}\left\{\frac{d^{n}}{d t^{n}}\left[g(t)-b_{-1}\left(t-t_{0}\right)^{-1}\right]\right\}_{t=0} .
$$

But these coefficients remain finite when $t_{0} \rightarrow 0$.

\section{Application to the Wedge Diffraction Problem}

The investigations outlined before will now be applied to a previously discussed problem. It was shown $[6,7]$ that the solution of the problem of the diffraction of plane waves by a wedge or corner is governed by the integral

with

$$
I(\alpha, \psi)=\int_{0}^{\infty} t^{-\frac{1}{2}} g(t) e^{-\gamma_{\rho} t} d t
$$

$$
g(t)=-(2 \alpha)^{-1} e^{-\gamma_{\rho}} \sin (\pi \psi / \alpha)(t+2)^{-\frac{1}{2}}
$$$$
\cdot\left\{\cosh \left[\pi / \alpha \log \left(1+t+\left(t^{2}+2 t\right)^{\frac{1}{2}}\right)\right]-\cos (\pi \psi / \alpha)\right\}^{-1} .
$$

Here $g(t)$ is analytic near the origin and the singularity nearest to the origin is a pole of the order one. The pole $t_{0}$ and its residue $b_{-1}$ are found to be

$$
\begin{gathered}
t_{o}=-2 \sin ^{2}\left(\frac{1}{2} \psi\right) \\
b_{-1}=-\pi^{-1} 2^{-\frac{1}{2}} e^{-\gamma \rho} \sin \left(\frac{1}{2} \psi\right) .
\end{gathered}
$$

The parameter $\psi$ in $(22)$ depends on the direction of the incident wave and on the direction of the point of observation such, that $\psi$ (and consequently $t_{0}$ ) tends to zero when one of the boundaries of geometric optics is approached. The asymptotic expansion of the integral (21) for large $\gamma \rho$ can then be obtained by means of (18), (19), and (20):

$$
\begin{aligned}
& I(\alpha, \psi) \approx \sum_{n=0}^{\infty} c_{n} \Gamma\left(n+\frac{1}{2}\right)(\gamma \rho)^{-n-\frac{1}{2}} \\
& \quad-\frac{1}{2} e^{-\gamma \rho \cos \left(\frac{1}{2} \psi\right)} \operatorname{Erfc}\left[(2 \gamma \rho)^{\frac{1}{2}} \sin \psi\right] .
\end{aligned}
$$

The coefficients $c_{n}$ are determined by (10) with

$g^{*}(t)=g(t)+\pi^{-1} 2^{-\frac{1}{2}} e^{-\gamma_{\rho}} \sin \left(\frac{1}{2} \psi\right)\left(t+2 \sin ^{2} \psi / 2\right)^{-1}$

and $g(t)$ is given by $(22)$. The function $g^{*}(t)$ fulfills the conditions (a) and (b) in the region $-\pi<\arg t<\pi$. The asymptotic expansion (25) holds therefore in $-3 \pi / 2<\arg (\gamma \rho)<3 \pi / 2$ and is valid also for $\psi=0$ (in which case all the $c_{n}$ vanish) and assumes in this case the correct value of (21)

$$
I(\alpha, 0)=-\frac{1}{2} e^{-\gamma_{\rho}} .
$$

The results produced here are equivalent to those in $[6,7]$.

\section{References}

[1] P. C. Clemmow, Some extensions to the method of integration by steepest descents, Quart. J. Mech. Appl. Math. (Oxford) 3, 241-256 (1950).

[2] E. T. Copson, Theory of a complex variable (Oxford, 1946).

[3] G. Doetsch, Handbuch der Laplace Transformation, Bd. 2, (Basel, 1955).

[4] G. Doetsch, Ein allgemeines Prinzip der asymptotischen Entwicklung, J. reine angew. Math. 167, 274-293 (1931).

[5] A. Erdelyi, Higher transcendental functions, vol. 1, (McGraw-Hill Book Co., Inc., New York, N.Y., 1953).

[6] F. Oberhettinger, On the diffraction and reflection of waves and of pulses by wedges and corners, J. Research NBS 61, 343-365 (1958).

[7] F. Oberhettinger, On asymptotic series for functions occurring in the theory of diffraction of waves by wedges, J. Math. Phys. 34, 245-255 (1956).

[8] H. Ott, Die Sattelpunktsmethode in der Ungebung eines Poles Mit Anwendung aud die Wellenoptik und Akustik, Ann. Phys, 43, 393-403 (1943).

[9] B. L. van der Waerder, On the method of saddle points, Appl. Sci. Research [B] 2, 33-45 (1951).

Washington, D.C.

(Paper 63B1-2) 